\title{
Macular Pigment Optical Density is Lower in Type 2 Diabetes, Compared with Type 1 Diabetes and Normal Controls.
}

James Loughman

Technological University Dublin, james.loughman@tudublin.ie

Grainne Scanlon

Technological University Dublin, grainne.scanlon@tudublin.ie

Paul Connell

Mater Misericordiae University Hospital, Dublin,

See next page for additional authors

Follow this and additional works at: https://arrow.tudublin.ie/otpomart

Part of the Ophthalmology Commons

\section{Recommended Citation}

Scanlon, G., Connell, P.P. \& RatzIFF, M. (2015). Macular pigment optical density is lower in Type 2 diabetes, compared with Type 1 diabetes and normal controls. Retina, vol. 35, no. 9, pg. 1808-16. doi:10.1097/ IAE.0000000000000551

This Article is brought to you for free and open access by ARROW@TU Dublin. It has been accepted for inclusion in Articles by an authorized administrator of ARROW@TU Dublin. For more information, please contact arrow.admin@tudublin.ie, aisling.coyne@tudublin.ie, gerard.connolly@tudublin.ie.

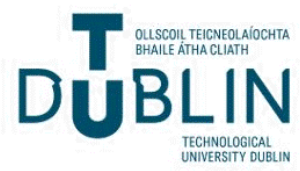




\section{Authors}

James Loughman, Grainne Scanlon, Paul Connell, Audrey Murphy, Karen O'Conner, Mathew Ratzlaff, and Brittany Foerg

This article is available at ARROW@TU Dublin: https://arrow.tudublin.ie/otpomart/46 
NUMBER 1 OF 1

\section{AUTHOR QUERIES}

DATE $\quad 2 / 12 / 2015$

JOB NAME RETINA

ARTICLE RETINA214-0493

QUERIES FOR AUTHORS Scanlon et al

\section{THIS QUERY FORM MUST BE RETURNED WITH ALL PROOFS FOR CORRECTIONS}

AU1) Please check the edits made to article title.

AU2) Please designate the affiliation $\S$ in author group details.

AU3) Please provide the department/unit (if any) in all affiliations except *.

AU4) Please confirm the conflicts of interest statement added.

AU5) Please spell out "CVD" in the sentence "Waist-to-height ratio has more recently been. ..."

AU6) Please provide publisher location for reference 29.

AU7) Please provide significance of bold values in Table 1. 


\title{
MACULAR PIGMENT OPTICAL DENSITY IS LOWER IN TYPE 2 DIABETES, COMPARED WITH TYPE 1 DIABETES AND NORMAL CONTROLS
}

\author{
GRAINNE SCANLON, MPHIL,* PAUL CONNELL, MD $\dagger$ MATTHEW RATZLAFF, BSC \\ BRITTANY FOERG, BSc $\neq$ DANIEL MCCARTNEY, PHD,$*$ AUDREY MURPHY, BSc,$\dagger$ \\ KAREN O'CONNOR, BSc, $\dagger$ JAMES LOUGHMAN, PHD*ף
}

Purpose: This study was designed to investigate the optical density of macular pigment in Type 1 and Type 2 diabetes subjects relative to normal controls.

Methods: One hundred and fifty subjects were recruited to the study and divided into one of the three study groups on the basis of their health status, as follows: Group 1: Healthy controls; Group 2: Type 1 diabetes; Group 3: Type 2 diabetes. Macular Pigment Optical Density, at $0.5^{\circ}$ of retinal eccentricity, was measured using customized heterochromatic flicker photometry. Dietary intake of macular carotenoids was quantified using a lutein and zeaxanthin food frequency questionnaire. Diabetes type, duration, medication, smoking habits, glycosylated hemoglobin $(\mathrm{HbA} 1 \mathrm{C})$, and serum lipid levels were recorded, whereas visual acuity, body mass index, and diabetic retinopathy grade were measured for each participant.

Results: One-way analysis of variance revealed a statistically significant difference in body mass index, age, high-density lipoprotein cholesterol and $\mathrm{HbA} 1 \mathrm{C}$ between the three groups $(P<0.01$ for all). Chi-square analysis revealed a statistically significant difference in diabetic retinopathy distribution $(P<0.01)$. None of these variables exhibited a statistically significant correlation with macular pigment optical density for any study group $(P>0.05$ for all). There was no difference in dietary carotenoid intake between groups. Macular pigment optical density was lower among Type 2 diabetes subjects $(0.33 \pm 0.21)$ compared with Type 1 diabetes $(0.49 \pm 0.23)$ and controls $(0.48 \pm 0.35)$. General linear model analysis, including age, body mass index, diabetes duration, diabetic retinopathy status, highdensity lipoprotein cholesterol, and $\mathrm{HbA1C}$ as covariates, revealed a statistically significant effect of diabetes type on macular pigment optical density $(\mathrm{F}=2.62 ; P=0.04)$.

Conclusion: Macular pigment optical density was statistically significantly lower in Type 2 diabetes compared with Type 1 diabetes and normal controls. Although body mass index was higher in the Type 2 diabetes group, the lower macular pigment optical density levels observed among Type 2 diabetes seem not to be attributable to differences in dietary carotenoid intake or to the specific presence of diabetes, diabetic control, duration, or diabetic retinopathy.

RETINA 00:1-9, 2015

$\mathrm{N}$ eurodegenerative diseases of the retina such as agerelated macular degeneration and diabetic retinopathy (DR) are leading causes of worldwide blindness. 1 Although the relationship between age-related macular degeneration and macular pigment optical density (MPOD) has been widely reported, ${ }^{2,3}$ only a small number of studies have focused on MPOD and carotenoid intake in diabetes mellitus, ${ }^{4,5}$ a condition similarly known to cause oxidative damage in the retina. ${ }^{6}$
Diabetes, a lifelong progressive disease, is the result of the body's inability to produce insulin or use insulin to its full potential and is characterized by high circulating glucose. ${ }^{7}$ Diabetic retinopathy represents the most common diabetic eye disease, and there is strong evidence that oxidative stress plays an important role in its development. ${ }^{6,7}$ Chronic hyperglycemia causes oxidative stress, ${ }^{7}$ which results in microvascular complications at the retina, where the neuronal elements 
responsible for vision are located. The relationship between hyperglycemia, changes in redox homeostasis, and oxidative stress are key events in the pathogenesis of DR. ${ }^{7}$ Oxidative stress is also involved in the initiation and progression of obesity and diabetes mellitus. $^{8}$ The links between Type 2 diabetes and obesity are firmly established. ${ }^{9}$ Type 2 diabetes now also affects a much younger population because of sedentary lifestyles and increases in calorific intake and accounts for more than $90 \%$ of all cases of diabetes. ${ }^{10}$

The development of ocular complications in diabetes is related to disease control and longevity. ${ }^{11-13}$ After 20 years with diabetes, $75 \%$ of patients will have some form of DR. ${ }^{12}$ Apart from good systemic control of blood sugar levels, hypertension, lipid profiles, and renal function, current treatment modalities for DR are limited to laser photocoagulation ${ }^{13}$ and/or intravitreal injections. ${ }^{14}$ These are effective modes of treatment but they also have their own limitations and side effects. New modalities should, therefore, be preventative in nature and ideally implemented long before overt clinical symptoms develop.

Macular pigment is believed to possess antioxidant properties and to limit retinal oxidative damage by absorbing harmful short-wavelength blue light. ${ }^{15}$ Macular pigment is highly concentrated at the central macula and is composed of the dietary hydroxycarotenoids lutein, zeaxanthin, and meso-zeaxanthin. ${ }^{16}$ These hydroxycarotenoids are found in the retina to the exclusion of all other 600 carotenoids found in nature. ${ }^{17}$ Concentrations of carotenoids in human serum and deposition of the macular carotenoids in the retina to form MP are highly variable and reflect not only dietary intake but also factors such as carotenoid chemistry, ${ }^{18}$ individual efficacy of absorption, ${ }^{19}$ fat intake, ${ }^{20}$ competition among carotenoids for absorption, ${ }^{21}$ cholesterol and lipoprotein status, ${ }^{22,23}$ metabolic status, ${ }^{4}$ body composition, and body fat/body mass index (BMI). ${ }^{21,2}$

The relationship between MP and diabetes is only now attracting research interest, possibly as a result of the outcomes of clinical trials, which demonstrate a protective effect of lutein and zeaxanthin supple-

From the *Department of Optometry, School of Physics, College of Sciences and Health, Dublin Institute of Technology, Dublin, Ireland; †Mater Misericordiae University Hospital, Dublin, Ireland; $\$$ School of Medicine and Medical Science, University College Dublin, Dublin, Ireland; §School of Biological Sciences, College of Sciences and Health, Dublin Institute of Technology, Dublin, AU2 Ireland; and IfAfrican Vision Research Institute, Faculty of Health

Sciences, University of KwaZulu Nalal, Durban, South Africa.

None of the authors have any financial/conflicting interests to disclose.

Reprint requests: James Loughman, PhD, Department of Optometry, School of Physics, College of Sciences and Health, Dublin Institute of Technology, Dublin 8, Ireland; e-mail: james. loughman@dit.ie mentation in another oxidative stress-related condition, age-related macular degeneration. ${ }^{2,3}$ One experimental study on diabetic rats demonstrated a reduction in retinal oxidative damage after carotenoid supplementation. ${ }^{25}$ Lutein and zeaxanthin intake has also been shown to improve macular edema in DR patients. ${ }^{5}$ These results suggest that MP supplementation has the potential to inhibit or delay the development of macular disease in patients with diabetes.

This study was designed to investigate the crosssectional relationship, if any, between diabetes and central MPOD and to explore the influence of potential explanatory factors, including glycosylated hemoglobin (HbA1C), serum lipid levels, BMI, and dietary carotenoid intake on any relationship that might be observed.

\section{Materials and Methods}

Diabetic and normal control subjects were recruited for this cross-sectional, case-control study at the Mater Misericordiae University Hospital in Dublin, Ireland. The study was approved by the Institutional Research Ethics Committee and adhered to the tenets of the Declaration of Helsinki. Informed consent was obtained from all subjects before enrollment and examination.

Generic inclusion criteria were as follows: subjects were to be older than 18 years, not having taken dietary supplements containing lutein, zeaxanthin or mesozeaxanthin over the 6-month period before the study, and $\log$ MAR visual acuity better than $0.2(6 / 9)$ in the study eye. For normal (control) subjects, exclusion criteria included any sign of retinal or ocular abnormality and the presence of Type 1 or Type 2 diabetes. Patients with diabetes were excluded if they exhibited signs of ocular comorbidity (e.g., glaucoma, cataract, age-related macular degeneration), had previously undergone any form of treatment for DR or maculopathy, or if they exhibited any signs of proliferative retinopathy or maculopathy. The study eye was selected using the eye with better visual acuity, or, in cases of equal acuity, the right eye was selected as standard.

Subjects were assigned into one of three study groups on the basis of their ocular health status and diabetes type as follows: Group 1: nondiabetic controls; Group 2: Type 1 diabetes; Group 3: Type 2 diabetes. The following diabetes-related information was recorded for each diabetic participant: diabetes duration (in years); diabetes type; DR grade (graded according to modified 2-field Early Treatment Diabetic Retinopathy Study protocol-grade range R0 M0 to R2 $\mathrm{M} 0$ ), and diabetes medication. Demographic information, including age, gender, and history of smoking 
(current smoker, exsmoker, and never smoker), was collected in relation to each subject.

\section{Procedures}

Macular pigment absorbs blue light and is optically undetectable at $6^{\circ}$ to $8^{\circ}$ eccentricity. ${ }^{26}$ In this study, MPOD was determined using the Macular Metrics Clinical Densitometer (Macular Metrics, Rehoboth, MA), which is based on the principle of heterochromatic flicker photometry. ${ }^{27}$ The basic measurement procedure involves presenting a small test stimulus that alternates between a measuring wavelength $(458 \mathrm{~nm})$, which is absorbed by macular pigment and a reference wavelength $(540 \mathrm{~nm})$ not absorbed by the pigment. The subject adjusts the intensity of the measuring wavelength $(458 \mathrm{~nm})$ luminance until there is minimum flicker (matching luminance). The luminance of the reference wavelength $(540 \mathrm{~nm})$ remains constant. The ratio of the amount of measuring wavelength $(458 \mathrm{~nm})$ light required to achieve the end point of matching luminance, or minimum flicker, for foveal and parafoveal readings is a measure of the amount of pigment present, and the logarithm of this ratio represents the optical density of MP.

Before using the Densitometer, all subjects were shown an explanatory video describing the method for recording null flicker matches. The measurement was conducted by a trained examiner, and all subjects were naive to the heterochromatic flicker photometry test. The MPOD measurement comprised the average of six readings (computed as the radiance value at which the subject reported null flicker) taken centrally at $0.5^{\circ}$ retinal eccentricity and again at $0.7^{\circ}$ retinal eccentricity. The target used for measuring MPOD at $0.5^{\circ}$ eccentricity was a solid disc of $0.5^{\circ}$ arc radius, and the parafoveal measurement was taken by asking the subject to fixate on a red light located precisely at $7^{\circ}$ from central fixation. Measurements were deemed reliable and acceptable only when the standard deviation of null flicker responses was below 0.1 .

A customized heterochromatic flicker photometry procedure was adopted, whereby flicker frequency was optimized for each participant before measurement of MPOD. ${ }^{28}$ The starting flicker was set at $10 \mathrm{~Hz}$ to 11 $\mathrm{Hz}$. If the subject was unable to find no/minimum flicker, this frequency was increased until no/minimal flicker could be identified. If the null one was too wide, the flicker frequency was reduced. The flicker frequency was adjusted till the subject found a narrow null zone.

Dietary carotenoid intake was estimated using the lutein/zeaxanthin questionnaire (Carotenoid \& Health Laboratory, Jean Mayer USDA Human Nutrition
Center on Aging, Tufts University, USA). ${ }^{29}$ Body weight was measured to the nearest $0.2 \mathrm{~kg}$ using a Seca Compact Digital Floor Scale 111, model 888 (Seca Limited), whereas height was measured to the nearest $0.5 \mathrm{~cm}$ using a collapsible "Leicester Height Measure" stadiometer (CMS Weighing Equipment). Body mass index was given as weight (in kilograms)/height (in square meters). Diabetes duration (in years), diabetes type, and laboratory results including $\mathrm{HbA1C}$ and serum lipid (high-density lipoprotein [HDL], lowdensity lipoprotein, total cholesterol, and triglycerides) levels of patients with diabetes were recorded for each subject.

\section{Statistical Analysis}

The statistical software package SPSS for Microsoft Windows (v.21.0; IBM Corp., Armonk, NY) was used for analysis. Data are presented as mean \pm standard deviation throughout. The data were tested for normality using the Kolmogorov-Smirnov test. One-way analysis of variance was used to test for differences in normally distributed study parameters between groups, whereas the Kruskal-Wallis test was used to test for differences in group medians in nonnormally distributed data. The chi-square test was used to compare categorical data across the three study groups. Pearson's product-moment correlation tests were performed to assess the relationship between MPOD and other study variables where appropriate. A general linear model approach was used to explore the relationship between diabetes type, other potential explanatory variables, and the dependent variable, MPOD. The level of statistical significance was set at the standard $P<0.05$.

\section{Results}

One hundred and fifty subjects were recruited to the study and divided into 1 of the 3 study groups on the basis of their ocular health status and diabetes type, as follows: Group 1: nondiabetic controls $(n=48$; male $=$ 20 , female $=28)$; Group 2: Type 1 diabetes $(n=34$; male $=17$, female $=17$ ); Group 3: Type 2 diabetes $(\mathrm{n}=68 ;$ male $=43$, female $=25)$.

Kolmogorov-Smirnov analysis revealed that BMI, total cholesterol, lutein intake, zeaxanthin intake, and MPOD data were normally distributed $(P>0.05$ for each), whereas all remaining variables exhibited nonnormal distributions. Accordingly, parametric tests were applied, where relevant, to normally distributed data, and nonparametric equivalent tests to nonnormally distributed data. A comparison of the demographic and clinical characteristics of each group is presented in Table 1. 
Table 1. Demographic and Clinical Findings According to Classification Group

\begin{tabular}{|c|c|c|c|c|}
\hline & Normal Controls & Type 1 Diabetes & Type 2 Diabetes & $P$ \\
\hline Sex & & & & $0.07^{*}$ \\
\hline Male & 20 & 17 & 43 & \\
\hline Female & 28 & 17 & 25 & \\
\hline Age, years & $52.48 \pm 16.03$ & $43.67 \pm 12.98$ & $62.67 \pm 11.31$ & $<0.01 \dagger$ \\
\hline $\mathrm{BMI}, \mathrm{kg} / \mathrm{m}^{2}$ & $27.7 \pm 4.8$ & $27.33 \pm 4.77$ & $31.44 \pm 6.16$ & $<0.01 \ddagger$ \\
\hline Smoking duration & 2.13 years & $2.35 \pm 0.60$ & $2.25 \pm 0.65$ & $0.33 \dagger$ \\
\hline DM duration, years & - & $22.73 \pm 10.85$ & $9.54 \pm 7.33$ & $<0.01 \dagger$ \\
\hline $\mathrm{HbA1c}$ & - & $7.9 \pm 1.08$ & $6.88 \pm 1.09$ & $<0.01 \dagger$ \\
\hline Triglycerides, mg/dL & - & $1.37 \pm 2.06$ & $1.55 \pm 0.85$ & $0.55 \dagger$ \\
\hline $\mathrm{HDL}, \mathrm{mg} / \mathrm{dL}$ & - & $1.52 \pm 0.46$ & $1.14 \pm 0.28$ & $<0.01 \dagger$ \\
\hline $\mathrm{LDL}, \mathrm{mg} / \mathrm{dL}$ & - & $2.37 \pm 0.70$ & $2.20 \pm 0.74$ & $0.15 \dagger$ \\
\hline Total cholesterol mg/dL & - & $4.31 \pm 0.82$ & $4.03 \pm 0.89$ & $0.14 \ddagger$ \\
\hline MPOD & $0.48 \pm 0.35$ & $0.49 \pm 0.23$ & $0.33 \pm 0.21$ & $<0.01 \ddagger$ \\
\hline Lutein intake, mg/day & $0.95 \pm 0.21$ & $1.06 \pm 1.29$ & $0.95 \pm 1.73$ & $0.93 \ddagger$ \\
\hline Zeaxanthin intake, mg/day & $0.11 \pm 0.12$ & $0.16 \pm 0.26$ & $0.12 \pm 0.25$ & $0.54 \ddagger$ \\
\hline Total lutein and zeaxanthin intake, mg/day & $1.07 \pm 1.25$ & $1.23 \pm 1.34$ & $1.07 \pm 1.83$ & $0.88 \ddagger$ \\
\hline Nonproliferative DR & - & $\begin{array}{c}\text { Yes }=23(68 \%) \\
\text { No }=11(32 \%)\end{array}$ & $\begin{array}{l}\text { Yes }=24(35 \%) \\
\text { No }=44(65 \%)\end{array}$ & $<0.01^{*}$ \\
\hline
\end{tabular}

${ }^{*}$ Chi-square test.

tKruskal-Wallis test.

‡One-way analysis of variance.

ANOVA, one-way analysis of variance; DM, diabetes mellitus; HbA1c, glycosylated hemoglobin level; LDL, low-density lipoprotein.

Post hoc analysis (Tukey test) revealed that MPOD was statistically significantly lower in Type 2 diabetes compared with both normal controls $(P=0.02)$ and Type 1 diabetes $(P=0.03)$, whereas the difference in MPOD between Type 1 diabetes and normal controls was not statistically significant $(P=0.99)$. The distribution of MPOD for each of the study groups is presented in Figure 1.

A general linear model analysis confirmed a significant effect of diabetes type on the dependent variable, MPOD $(P=0.04)$ but no effect of potentially confounding variables including age, BMI, DR status, HDL cholesterol, or HbA1c on MPOD $(P=0.16-0.74)$.

The distribution of MPOD according to DR status, which demonstrated no influence on MPOD in the general linear model analysis $(P=0.45)$, is presented

F2 in Figure 2.

Body mass index was statistically significantly higher in Type 2 diabetes compared with normal controls $(P<$ $0.01)$ and Type 1 diabetes $(P<0.01)$, whereas the difference in BMI between Type 1 diabetes and normal controls was not statistically significant $(P=0.75)$. Body mass index, however, including controlling for confounding was not statistically significantly correlated with MPOD ( $\mathrm{r}=0.08, P=0.51)$. The distribution of BMI

\section{Discussion}

This study demonstrates that MPOD levels are significantly lower in Type 2 diabetes, when compared

with Type 1 diabetes, which represents an entirely novel finding. This observation is significant given that similar dietary carotenoid intake was observed among the study groups and in the context of shorter diabetes duration among the Type 2 diabetes subjects. Similarly, the presence of nonproliferative DR seems not to influence MPOD levels, which are, surprisingly, slightly higher on average in those with retinopathy compared with those without retinopathy, although lower in both diabetic groups compared with normal controls.

The study further demonstrates that the MPOD levels in Type 2 diabetes are significantly lower than in normal controls, a finding, which is in general agreement with those of previous investigations that have explored the relationship between MPOD and diabetes. It has been shown, for example, that Type 2 diabetic subjects had lower MPOD when compared with nondiabetic controls, while Type 2 diabetes with mild nonproliferative retinopathy exhibited similar MPOD levels to Type 2 diabetes without retinopathy. ${ }^{4}$ In addition, serum lutein and zeaxanthin concentrations, which have been shown to be positively and significantly related to MPOD in normal subjects, ${ }^{30}$ have been shown to be significantly lower in diabetes compared with normal subjects. ${ }^{5}$ In more advanced cases of diabetic eye disease, subjects with Grade 2 maculopathy were found to have significantly lower MPOD than those without maculopathy. ${ }^{31}$ The apparent relationship between low MP levels and increasing severity of maculopathy prompted those study investigators to implicate oxidative stress as a causative factor, a concept that merits further discussion here. ${ }^{31}$ 


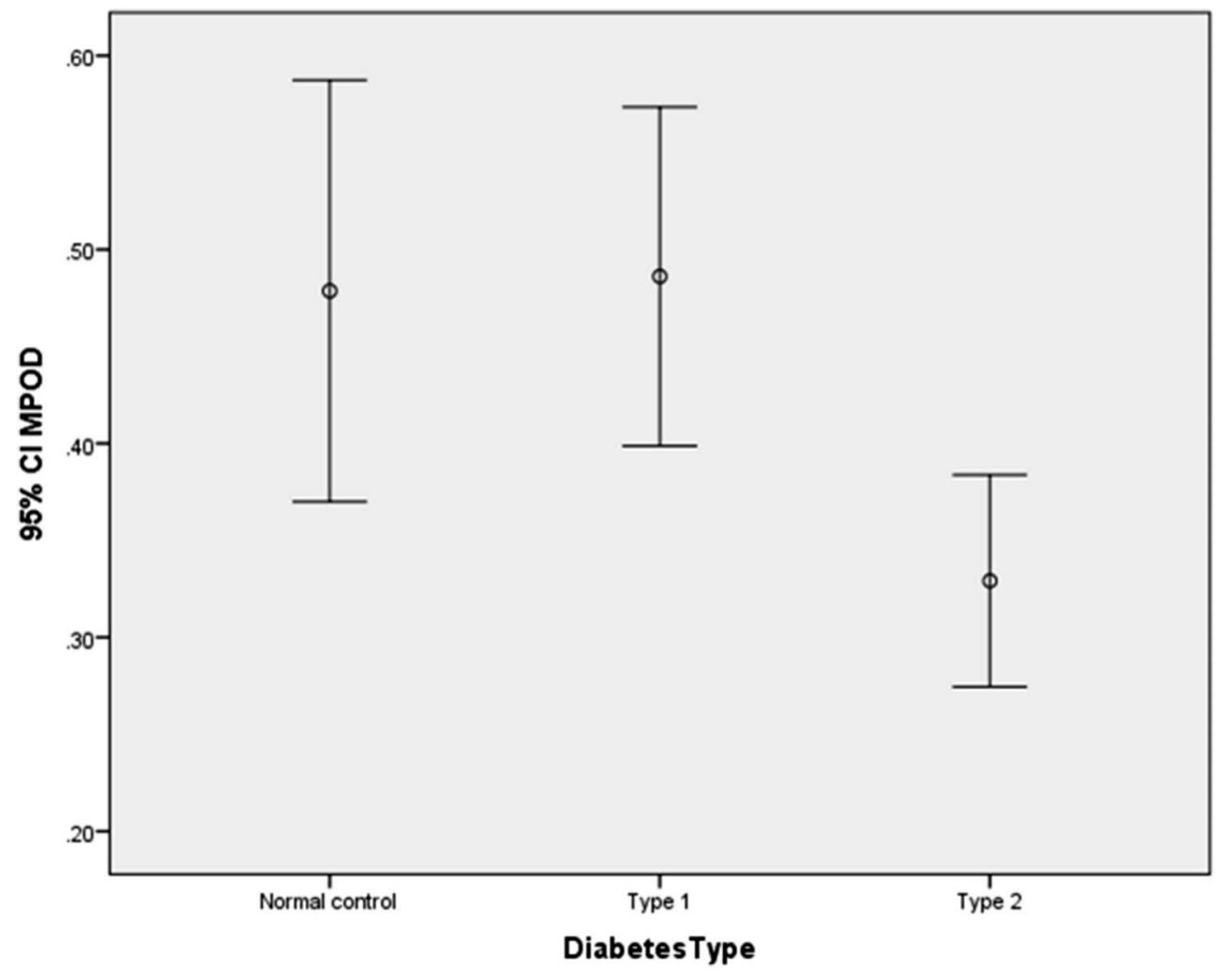

Fig. 1. Macular pigment optical density distribution (mean $\pm 95 \%$ confidence intervals) according to diabetes status, illustrating the substantially lower MPOD levels among Type 2 diabetic subjects.
Macular pigment optical density has a slow biological turnover, as it reflects the local balance between prooxidant stresses and antioxidant defenses in the retina. ${ }^{30}$ The retina is particularly susceptible to oxidative stress because of its high oxygen consumption, high proportion of polyunsaturated fatty acids, and exposure to visible and short-wavelength blue light. ${ }^{32}$ There is also considerable evidence that hyperglycemia results in the generation of reactive oxygen species, ultimately leading to increased oxidative stress in the retina, ${ }^{6,7}$ which may play an important role in the etiology of diabetic complications. Poor metabolic control and longer duration of diabetes are directly linked to the prevalence of $\mathrm{DR},{ }^{11}$ whereas oxidative stress can be significantly reduced with aggressive control of hyperglycemia. ${ }^{33}$ We found no association, however, between MPOD and either $\mathrm{HbAlc}$ or diabetes duration in this study. Paradoxically, despite their lower MPOD, metabolic control appeared better, diabetes duration was shorter, and DR prevalence was lower for Type 2 compared with Type 1 diabetic subjects. Although HbAlc has previously been shown to be inversely and significantly related to MPOD among a smaller sample of Type 2 diabetic subjects, ${ }^{4}$ our findings imply that lower MPOD in Type 2 diabetes is not attributable to the diabetic condition, duration, or level of control, and suggests a need to explore alternative explanatory causes.
Although BMI measures do not provide a precise indicator of adiposity, it is likely that the statistically significantly higher BMI levels observed in the Type 2 diabetes group here, which averaged above WHOdefined BMI levels indicative of obesity (BMI > 30), ${ }^{34}$ are reflective of excess adiposity in this group compared with the normal control and Type 1 diabetes groups. To date, there has been very little consensus on the relationship between types of obesity and MPOD. One study reported a lack of an association between MPOD and various obesity indices, including waist circumference, waist-to-hip ratio, and BMI, in a South-Indian population. ${ }^{35}$ Conversely, other studies have shown significant inverse relationships between body fat and MPOD in humans ${ }^{20,21,24}$; however, these relationships differed between men $^{20}$ and women. ${ }^{21}$

Lutein and zeaxanthin are known to accumulate in adipose tissue, ${ }^{36}$ and lower MPOD values have also been reported among individuals exhibiting body fat measuring greater than $27 \%$, when compared with those with lower body fat. ${ }^{24}$ Higher body fat percentage even within relatively healthy limits is associated with lower tissue lutein and zeaxanthin status ${ }^{37}$ and renders the macular carotenoids less available to retinal tissue. ${ }^{38}$ Distribution of body fat in the body is also important, and it has been shown that lutein/zeaxanthin concentrations in adipose tissue differ according 
Fig. 2. The distribution of MPOD (mean $\pm 95 \%$ confidence intervals) according to DR status is illustrated. There is no significant difference in MPOD between patients with or without retinopathy indicating that retinopathy status seems not to be the main driver in influencing MP levels.

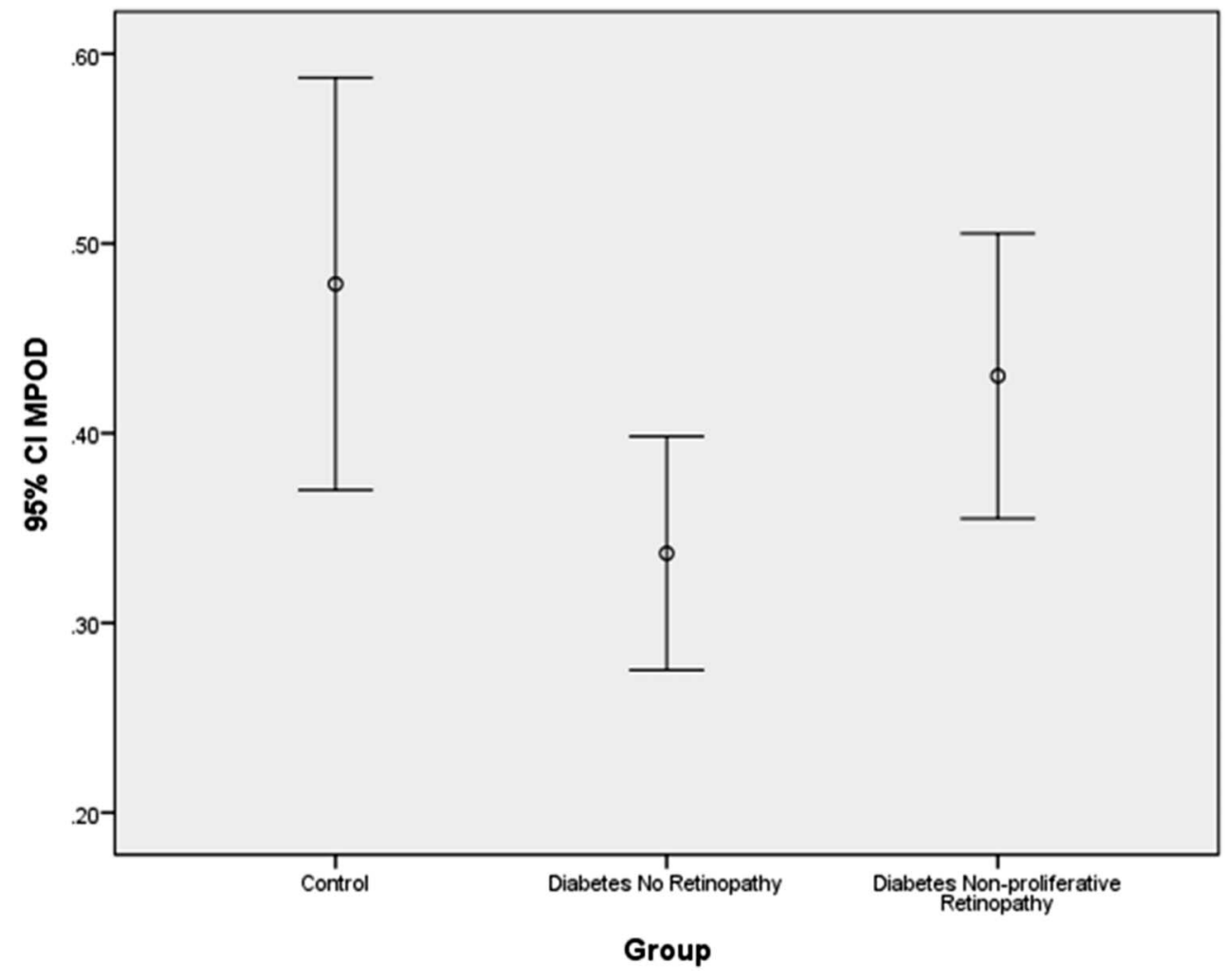

to body site, with levels demonstrably higher in abdominal fat than in the gluteofemoral fat depot. ${ }^{39}$ Interestingly, abdominal obesity is now considered an important risk factor and predictive indicator for the development of Type 2 diabetes and cardiovascuAU5 lar disease. ${ }^{40}$ Waist-to-height ratio has more recently been shown to have superior discriminatory power for detecting cardiovascular risk factors in both sexes ${ }^{41}$ and should be considered as a screening tool for diabetes, hypertension, and CVD in men and women. ${ }^{41}$ Future studies investigating the link between MPOD and obesity should, therefore, encompass more refined methods of body fat assessment including bioelectrical impedance analysis and waist-to-height ratio ${ }^{41}$ because BMI measurements alone may not be adequate to provide a true indication of adiposity, and this could explain the lack of an association between MPOD and BMI in our study.

Diets, which are high in fat and low in carotenoids, have been linked with increased oxidative stress. ${ }^{42}$ Obesity is also independently associated with increased oxidative stress, ${ }^{42-44}$ and an increased BMI has also been shown to be associated with increased risk of DNA damage due to oxidative stress. ${ }^{45}$ Serum 8-hydroxy 20-deoxy-guanosine (8-OhdG), for example, a known sensitive marker of oxidative DNA damage and of total systemic oxidative stress in vivo, has been shown to be positively correlated with BMI in people with Type 2 diabetes mellitus. ${ }^{46}$

Adipose tissue produces bioactive substances called adipokines, which induce the production of reactive oxygen species by a combination of mechanisms including mitochondrial and peroxisomal oxidation of fatty acids and overconsumption of oxygen, ${ }^{43,44}$ thereby initiate a process of oxidative stress ${ }^{43,44}$ Recent studies have highlighted the role of increased abdominal fat mass as a key driver of inflammation in Type 2 diabetes, ${ }^{47}$ a state closely associated with increased oxidative stress ${ }^{43,44}$ and macrovascular disease. ${ }^{47}$ Chronic inflammation induces changes in metabolic pathways and is believed to play a significant role in the progression from obesity to Type 2 diabetes. ${ }^{48,49}$

Despite the lack of any direct association between BMI and MPOD observed herein, it is plausible to suggest that 1) more refined methods of body fat assessment such as bioelectrical impedance analysis 50 and waist-to-height ratio ${ }^{41}$ might reveal an association that could explain the lower MPOD values observed in Type 2 diabetic subjects here and 2) that the combined effect of increased competition for lutein/zeaxanthin deposition and increased inflammation and oxidative stress levels in association with higher BMI/body fat, might explain, at least in part, the lower MPOD levels observed in the Type 2 diabetes group. 


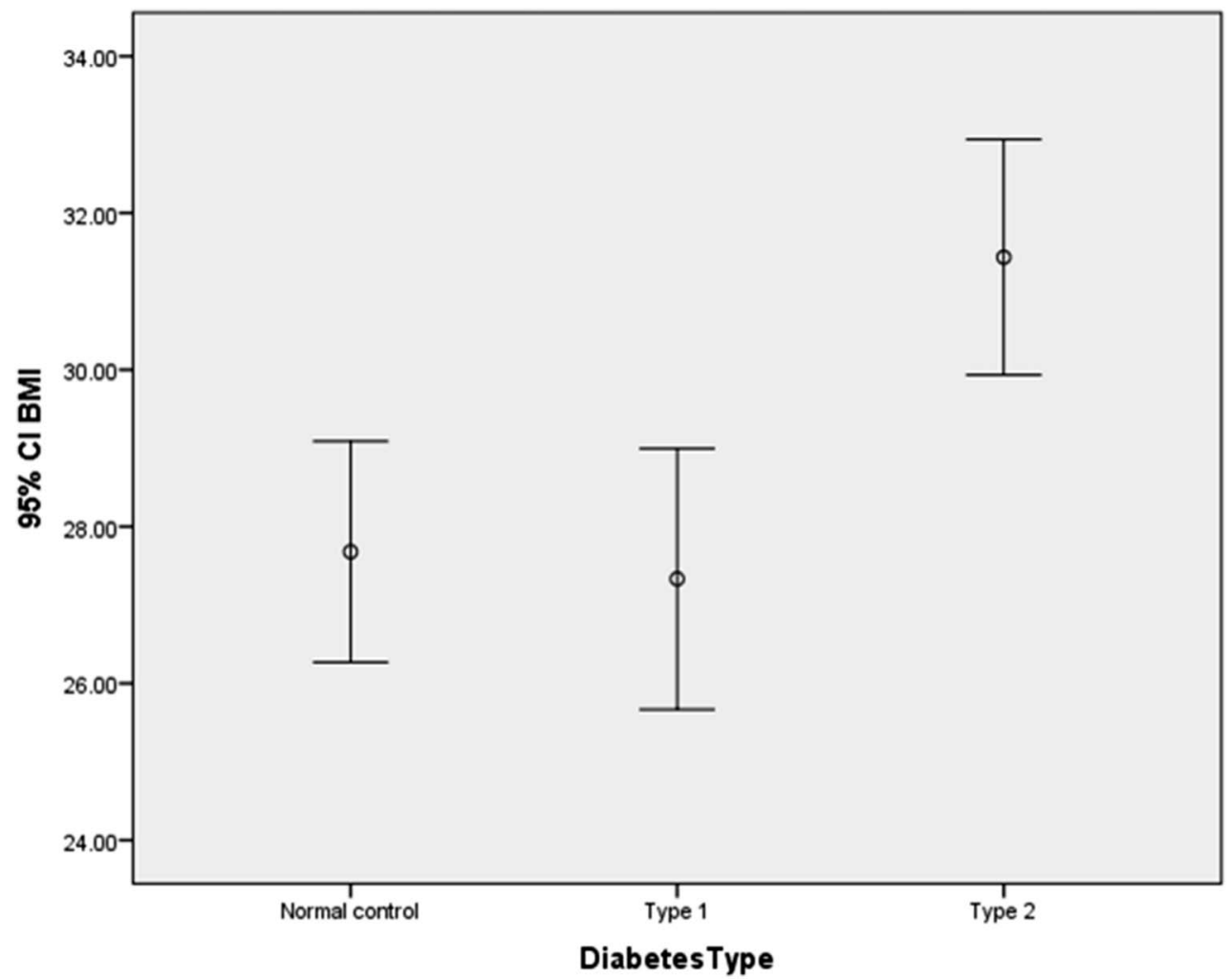

Fig. 3. Body mass index distribution (mean $\pm 95 \%$ confidence intervals) according to diabetes status, illustrating substantially higher BMI levels among Type 2 diabetic subjects.
High-density lipoprotein differences observed between Type 1 and Type 2 diabetes could also contribute to the lower MP levels observed in the Type 2 diabetes group. Macular pigment carotenoids are primarily transported by HDL in plasma. ${ }^{22,23}$ It has been suggested that mechanisms governing the retinal capture and/or stabilization of these carotenoids in the retina may be subject to HDL influence, by affecting receptor-mediated uptake of these carotenoids from serum. ${ }^{23}$ High-density lipoprotein deficiencies are associated with lutein and zeaxanthin tissue deficiencies, most notably in the retina. ${ }^{22}$ Furthermore, it has been suggested that individuals exhibiting elevated serum triglyceride concentrations and concurrently reduced serum HDL concentrations may have a related and reduced capacity to transport lutein in serum. ${ }^{23}$ Such features are very characteristic of Type 2 but not Type 1 diabetes. ${ }^{51}$ Lipoprotein profile is adversely affected by insulin resistance and might mechanistically explain (by eliciting a reduced capacity to transport macular carotenoids), why Type 2 diabetes is associated with lower MP levels compared with Type 1 diabetes where insulin resistance is much less prominent. A more comprehensive investigation into the association between lipoprotein profile, MPOD, and dietary carotenoid intake among diabetic subjects is therefore warranted.

Average macular carotenoid intake was similar for the three study groups $(P=0.88)$ and at levels that are consistent with the lower limits of average daily carotenoid intake, (ranges from 1.1 to $1.6 \mathrm{mg} / \mathrm{day}$ ). ${ }^{30}$ Although dietary intake is the primary driver of tissue carotenoid levels, ${ }^{15}$ the relationship between diet and deposition of these pigments in the retina is moderated by a number of factors. Retinal capture and/or stabilization of these carotenoids in the macula, may, for example, be subject to influence by obesity-induced inflammation and oxidative stress, ${ }^{48,49}$ competition between adipose and retinal tissue for the dietary carotenoids, ${ }^{21,24,37}$ impaired transport of circulating lutein/zeaxanthin, ${ }^{22,23}$ and/or genetic influence, ${ }^{52}$ which may help to explain the significant effect of Type 2 diabetes on MPOD.

Limitations to this cross-sectional case control study include the use of a dietary questionnaire designed for an American population among European participants, lack of serum carotenoid analysis, lack of detailed body fat analysis, and the absence of other clinical parameters such as inflammatory markers. Further research is needed in the diabetic population to uncover the relationship between disease progression, BMI including waist-to-height ratio, body fat composition, serum cholesterol, inflammatory status, and dietary carotenoid intake, as well as the mechanism of retinal damage in the presence of low MPOD. The effects of the adiposity, insulin resistance, inflammation, and HDL suppression characteristics that 
differentiate Type 2 from Type 1 diabetes on MPOD should be particularly emphasized.

A longitudinal study, comparing MPOD in Type 1 and Type 2 diabetes with parameters such as lipoprotein profile (including low-density lipoprotein, HDL, and triglycerides), ${ }^{22,23}$ obesity indices (including waist-toheight, bioelectrical impedance analysis, BMI, waist circumference, and waist-to-hip measurements), ${ }^{41}$ and dietary intake of lutein and zeaxanthin, ${ }^{31}$ would certainly provide a more holistic understanding of the relationship between MPOD and BMI in Type 2 diabetic patients over time. Certain features such as obesity, ${ }^{8}$ low HDL, and raised triglycerides are characteristic of Type 2 but not Type 1 diabetes, ${ }^{51}$ and these characteristics may indeed affect the transport, uptake, and stabilization of these carotenoids in the retina and would, therefore, warrant further investigation.

Key words: customized heterochromatic flicker photometry, diabetes mellitus, diabetic retinopathy, glycosylated hemoglobin, lutein, macular pigment optical density, meso-zeaxanthin, zeaxanthin.

\section{References}

1. Taylor HR, Keeffe JE. World blindness: a 21st century perspective. Br J Ophthalmol 2001;85:261-266.

2. Bernstein PS, Deloris FC, Richer S, et al. The value of measurement of macular carotenoid pigment optical densities and distributions in age-related macular degeneration and other retinal disorders. Vision Res 2010;50:716-728.

3. Snodderly DM. Evidence for protection against age-related macular degeneration by carotenoids and antioxidant vitamins. Am J Clin Nutr 1995;62:1448S-1461S.

4. Lima VC, Rosen RB, Maia M, et al. Macular pigment optical density measured by dual-wavelength autofluorescence imaging in diabetic and nondiabetic patients: a comparative study. Invest Ophthalmol Vis Sci 2010;51: 5840-5845.

5. $\mathrm{Hu} \mathrm{BJ}, \mathrm{Hu}$ YN, Lin S, et al. Application of Lutein and Zeaxanthin in nonproliferative diabetic retinopathy. Int J Ophthalmol 2011;4:303-306.

6. Van Reyk DM, Gillies MC, Davies MJ. The retina: oxidative stress and diabetes. Redox Rep 2003;8:187-192.

7. Koworlu RA, Chan PS. Oxidative stress and diabetic retinopathy. Exp Diabetes Res 2007;2007:43603.

8. Vîrgolici B, Mohora M, Stoian I, et al. A comparative oxidative stress study - obesity with and without diabetes mellitus. Rom J Intern Med 2005;43:261-268.

9. Freemantle N, Holmes J, Hockey A, Kumar S. How strong is the association between abdominal obesity and the incidence of type 2 diabetes? Int J Clin Pract 2008;62:1391-1396.

10. Hu FB. Globalization of diabetes. The role of diet, lifestyle and genes. Diabetes Care 2011;34:1249-1257.

11. Klein R, Klein BE, Moss SE. Relation of glycemic control to diabetic microvascular complications in diabetes mellitus. Ann Intern Med 1996;124:90-96.

12. Barcelo A, Aedo C, Rajpathak S, et al. The cost of diabetes in Latin America and the Caribbean. Bull World Health Organ 2003;81:19-27.
13. Gurelik G, Coney JM, Zakov ZN. Binocular indirect panretinal laser photocoagulation for the treatment of proliferative diabetic retinopathy. Ophthalmic Surg Lasers Imaging 2004;35:94-102.

14. Salam A, Mathew R, Sivaprasad S. Treatment of proliferative diabetic retinopathy with anti-VEGF agents. Acta Ophthalmol 2011;89:405-411.

15. Beatty S, Boulton ME, Henson DB. Macular pigment and agerelated macular degeneration. Br J Ophthalmol 1999;83:867-877.

16. Bone RA, Landrum JT, Hime GW, et al. Stereochemistry of the human macular carotenoids. Invest Ophthalmol Vis Sci 1993;34:2033-2040.

17. Landrum JT, Bone RA. Lutein, zeaxanthin and the macular pigment. Arch Biochem Biophys 2001;385:28-40.

18. Erdman JW Jr.. Variable bioavailability of carotenoids from vegetables. Am J Clin Nutr 1999;70:179-180.

19. Schupp C, Olano-Martin E, Gerth C, et al. Lutein, zeaxanthin, macular pigment, and visual function in adult cystic fibrosis patients. Am J Clin Nutr 2004;79:1045-1052.

20. Nolan JM, Donovan OO, Kavanagh H, et al. Macular pigment and percentage of body fat. Invest Ophthalmol Vis Sci 2004; 45:3940-3950.

21. Johnson EJ, Hammond BR, Yeum KJ, et al. Relation among serum and tissue concentrations of lutein and zeaxanthin and macular pigment density. Am J Clin Nutr 2000;71:1555-1562.

22. Connor WE, Duell PB, Kean R, Wang Y. The prime role of HDL to transport lutein into the retina: evidence from HDLdeficient WHAM chicks having a mutant ABCA1 transporter. Invest Ophthalmol Vis Sci 2007;48:4226-4231.

23. Loane E, Nolan JM, Beatty S. The respective relationships between lipoprotein profile, macular pigment optical density and serum concentrations of lutein and zeaxanthin. Invest Ophthalmol Vis Sci 2010;51:5897-5905.

24. Hammond BR, Ciulla TA, Snodderly DM. Macular pigment density is reduced in obese subjects. Invest Ophthalmol Vis Sci 2002;43:47-50.

25. Kowloru RA, Menon B, Gierhart DL. Beneficial effect of zeaxanthin on retinal metabolic abnormalities in diabetic rats. Invest Ophthalmol Vis Sci 2008;49;1645-1651.

26. Snodderly DM, Auran JD, Delori FC. The macular pigment 11. Spatial distribution in primate retinas. Invest Ophthalmol Vis Sci 1984;25:674-685.

27. Wooten BR, Hammond BR, Land RI, Snodderly DM. A practical method for measuring macular pigment optical density. Invest Ophthalmol Vis Sci 1999;40:2481-2489.

28. Stringham JM, Hammond BR, Nolan JM, et al. The utility of using customised heterochromatic flicker photometry (cHFP) to measure macular pigment in patients with age-related macular degeneration. Exp Eye Res 2008;87:445-453.

29. Johnson E, Rasmussen H, Burnett DJ. LZQ: A Dietary Questionnaire for Lutein and Zeaxanthin. Tufts University; 2009.

30. Nolan JM, Stack J, O'Connell E, Beatty S. The relationships between macular pigment optical density and its constituent carotenoids in diet and serum. Invest Ophthalmol Vis Sci 2007;48:571-582.

31. Davies NP, Morland AB. Colour matching in diabetes: optical density of the crystalline lens and macular pigments. Invest Ophthalmol Vis Sci 2002;43:281-289.

32. Beatty S, Koh H, Phil M, et al. The role of oxidative stress in the pathogenesis of age-related macular degeneration. Surv Ophthalmol 2000;45:115-134.

33. Davi G, Ciabattoni G, Consoli A, et al. In vivo formation of 8-iso-prostaglandin $\mathrm{f} 2 \mathrm{a}$ and platelet activation in diabetes mellitus effects of improved metabolic control and vitamin E supplementation. Circulation 1999;99:224-229. 
34. Sikaris KA. The clinical biochemistry of obesity. Clin Biochem Rev 2004;25:165-181.

35. Gupta A, Raman R, Biswas S, et al. Association between various types of obesity and macular pigment optical density. Eye (Lond) 2012;26:259-266.

36. Thompson LR, Toyoda Y, Langner A, et al. Elevated retinal zeaxanthin and prevention of light-induced photoreceptor cell death in quail. Invest Ophthalmol Vis Sci 2002;43:3538-3549.

37. Bovier ER, Lewis RD, Hammond BR Jr.. The relationship between lutein and zeaxanthin status and body fat. Nutrients 2013;5:750-757.

38. Gruber M, Chappell R, Millen A, et al. Correlates of serum lutein and zeaxanthin: findings from the Third National Health and Nutrition Examination Survey. J Nutr 2004;134:2387-2394.

39. Chung HY, Ferreira AL, Epstein S, et al. Site-specific concentrations of carotenoids in adipose tissue: relations with dietary and serum carotenoid concentrations in healthy adults. Am J Clin Nutr 2009;90:533-539.

40. Siren R, Eriksson JG, Vanhanen H. Waist circumference a good indicator of future risk for type 2 diabetes and cardiovascular disease. BMC Public Health 2012;12:631.

41. Ashwell M, Gunn P, Gibson S. Waist-to-height ratio is better screening tool than waist circumference and BMI for adult cardiometabolic risk factors: systematic review and meta-analysis. Obes Rev 2012;13:275-286.

42. Savini I, Catani MV, Evangelista D, et al. Obesity-associated oxidative stress: strategies finalized to improve redox state. Int J Mol Sci 2013;14:10497-10538.

43. Fernández-Sánchez A, Madrigal-Santillán E, Bautista M, et al. Inflammation, oxidative stress, and obesity. Int J Mol Sci 2011; 12:3117-3132.
44. Bondia-Pons I, Ryan L, Martinez JA. Oxidative stress and inflammation interactions in human obesity. J Physiol Biochem 2012;68:701-711.

45. Hofer T, Karlsson HL, Moller L. DNA oxidative damage and strand breaks in young healthy individuals: a gender difference and the role of life style factors. Free Radic Res 2006;40: 707-714.

46. Al-Aubaidy HA, Jelinek HF. Oxidative DNA damage and obesity in type 2 diabetes mellitus. Eur J Endocrinol 2011; 164:899-904.

47. Dahlén EM, Tengblad A, Länne T, et al. Abdominal obesity and low-grade systemic inflammation as markers of subclinical organ damage in type 2 diabetes. Diabetes Metab 2014; 40:76-81.

48. Kwon H, Pessin JE. Adipokines mediate inflammation and insulin resistance. Front Endocrinol (Lausanne) 2013; $4: 71$.

49. Luft VC, Schmidt MI, Pankow JS, et al. Chronic inflammation role in the obesity-diabetes association: a case-cohort study. Diabetol Metab Syndr 2013;5:31.

50. Meeuwsen S, Horgan GW, Elia M. The relationship between BMI and percent body fat, measured by bioelectrical impedance, in a large adult sample is curvilinear and influenced by age and sex. Clin Nutr 2010;29:560-566.

51. Vergès BL. Dyslipidaemia in diabetes mellitus. Review of the main lipoprotein abnormalities and their consequences on the development of atherogenesis. Diabetes Metab 1999;25(suppl 3):32-40.

52. Hammond BJ, Fuld K, Curran-Celentano J. Macular pigment density in monozygotic twins. Invest Ophthalmol Vis Sci 1995;36:2531-2541. 